\title{
Thermalization time scales for WIMP capture by the Sun
}

\author{
Axel Widmark* \\ Stockholm University \\ E-mail: axel.widmarkefysik.su.se
}

This work concerns the process of dark matter capture by the Sun, under the assumption of a Weakly Interacting Massive Particle (WIMP). Galactic halo WIMPs can collide with atomic nuclei in the solar interior. If they lose enough kinetic energy in such a collision, they will be bound in orbit. With further collisions, they will settle to thermal equilibrium in the Sun's core, where they can annihilate to produce a flux of neutrinos. Observing such a neutrino signal would be a smoking gun of indirect dark matter particle detection. This work aims to examine the process of thermalization for captured WIMPs, using Monte-Carlo integration of WIMP trajectories, given different WIMP-nucleon interaction operators of a non-relativistic effective field theory. The density of thermalized WIMPs are found to adhere to a thermal profile. With the exceptions of some fine-tuned cases, the thermalization time is significantly shorter than the age of the solar system.

35th International Cosmic Ray Conference

10-20 July, 2017

Bexco, Busan, Korea

${ }^{*}$ Speaker. 


\section{Introduction}

These proceedings are based on [1]; see this reference for further information.

Observational evidence of vast physical scales indicate that dark matter constitutes a majority of the Universe's matter content $[2,3]$. One of the most favored dark matter particle candidates is the Weakly Interacting Massive Particle (WIMP), whose existence can be inferred indirectly by observing its decay products. Halo WIMPs traversing the solar interior can interact with atomic nuclei and become bound by the Sun's gravitational field. With time, a captured WIMP will down-scatter until it reaches thermal equilibrium. Over time, a concentration of thermalized WIMPs builds up in the solar core, where they can annihilate in pairs to produce a flux of neutrinos, differentiable from fusion neutrinos due to their higher energy [4, 5]. In recent years, the framework of non-relativistic effective field theories has been applied to dark matter particle experiments; such studies pertaining to dark matter capture by the Sun can be found in $[6,7,8,9,10]$.

This work studies the thermalization process for WIMP capture by the Sun, in the model independent framework of a non-relativistic effective field theory. In particular, the aim is to find the median thermalization time, which is the time it takes for a WIMP bound in orbit to thermalize to core temperature. This time is most often neglected and approximated as instantaneous. Furthermore, this work also studies the density distribution of thermalized WIMPs, which is often assumed to follow a thermal profile. A departure from these assumptions on the WIMP distribution in the Sun can have a direct effect on the annihilation rate and neutrino flux.

\section{Effective field theory of WIMP-nucleon interactions}

WIMP interactions with atomic nuclei are modelled in a framework of non-relativistic effective field theory. The WIMP-nucleon quantum operators that are allowed in such a framework are

$$
\mathbb{1}_{\chi N}, \quad \hat{\mathbf{S}}_{\chi}, \quad \hat{\mathbf{S}}_{N}, \quad i \hat{\mathbf{q}}, \quad \hat{\mathbf{v}}^{\perp},
$$

where index $\chi(N)$ refers to a WIMP (nucleon), $\mathbf{S}$ denotes a spin vector, $\mathbf{q}$ is the transferred momentum of the collision, and $\mathbf{v}^{\perp} \equiv \mathbf{v}+\mathbf{q} / 2 \mu_{N}$ is the transverse velocity as given by collisional velocity $\mathbf{v}$ and the reduced mass of the WIMP-nucleon system $\mu_{N}$.

Assuming a heavy force mediating particle of spin 1 or less, the 14 possible leading order operators that can be constructed from the above building blocks are visible in table 1. As there are two types of nucleons, the number of degrees of freedom for possible WIMP-nucleon interactions in this framework amounts to a total number of 28. In this work, the chosen basis is one of isospin; for isoscalar coupling WIMPs interact with protons and neutrons in exactly the same manner, for isovector coupling the proton and neutron interactions have opposite signs.

This leads to a differential WIMP-nucleus cross, $\mathrm{d} \sigma\left(E_{r}, w^{2}\right) / \mathrm{d} E_{r}$, dependent on the recoil energy $E_{r}$ and the collisional velocity $w[9,11]$, unique for every isotope. In this work, I consider the 16 most abundant isotopes in the Sun: $\mathrm{H},{ }^{4} \mathrm{He},{ }^{16} \mathrm{O},{ }^{12} \mathrm{C},{ }^{20} \mathrm{Ne},{ }^{14} \mathrm{~N},{ }^{56} \mathrm{Fe},{ }^{28} \mathrm{Si},{ }^{24} \mathrm{Mg},{ }^{32} \mathrm{~S},{ }^{3} \mathrm{He}$, ${ }^{59} \mathrm{Ni},{ }^{40} \mathrm{Ar},{ }^{40} \mathrm{Ca},{ }^{27} \mathrm{Al},{ }^{23} \mathrm{Na}$. 


\begin{tabular}{ll}
\hline$\hat{\mathscr{O}}_{1}=\mathbb{1}_{\chi N}$ & $\hat{\mathscr{O}}_{9}=i \hat{\mathbf{S}}_{\chi} \cdot\left(\hat{\mathbf{S}}_{N} \times \frac{\hat{\mathbf{q}}}{m_{N}}\right)$ \\
$\hat{\mathscr{O}}_{3}=i \hat{\mathbf{S}}_{N} \cdot\left(\frac{\hat{\mathbf{q}}}{m_{N}} \times \hat{\mathbf{v}}^{\perp}\right)$ & $\hat{\mathscr{O}}_{10}=i \hat{\mathbf{S}}_{N} \cdot \frac{\hat{\mathbf{q}}}{m_{N}}$ \\
$\hat{\mathscr{O}}_{4}=\hat{\mathbf{S}}_{\chi} \cdot \hat{\mathbf{S}}_{N}$ & $\hat{\mathscr{O}}_{11}=i \hat{\mathbf{S}}_{\chi} \cdot \frac{\hat{\mathbf{q}}}{m_{N}}$ \\
$\hat{\mathscr{O}}_{5}=i \hat{\mathbf{S}}_{\chi} \cdot\left(\frac{\hat{\mathbf{q}}}{m_{N}} \times \hat{\mathbf{v}}^{\perp}\right)$ & $\hat{\mathscr{O}}_{12}=\hat{\mathbf{S}}_{\chi} \cdot\left(\hat{\mathbf{S}}_{N} \times \hat{\mathbf{v}}^{\perp}\right)$ \\
$\hat{\mathscr{O}}_{6}=\left(\hat{\mathbf{S}}_{\chi} \cdot \frac{\hat{\mathbf{q}}}{m_{N}}\right)\left(\hat{\mathbf{S}}_{N} \cdot \frac{\hat{\mathbf{q}}}{m_{N}}\right)$ & $\hat{\mathscr{O}}_{13}=i\left(\hat{\mathbf{S}}_{\chi} \cdot \hat{\mathbf{v}}^{\perp}\right)\left(\hat{\mathbf{S}}_{N} \cdot \frac{\hat{\mathbf{q}}}{m_{N}}\right)$ \\
$\hat{\mathscr{O}}_{7}=\hat{\mathbf{S}}_{N} \cdot \hat{\mathbf{v}}^{\perp}$ & $\hat{\mathscr{O}}_{14}=i\left(\hat{\mathbf{S}}_{\chi} \cdot \frac{\hat{\mathbf{q}}}{m_{N}}\right)\left(\hat{\mathbf{S}}_{N} \cdot \hat{\mathbf{v}}^{\perp}\right)$ \\
$\hat{\mathscr{O}}_{8}=\hat{\mathbf{S}}_{\chi} \cdot \hat{\mathbf{v}}^{\perp}$ & $\hat{\mathscr{O}}_{15}=-\left(\hat{\mathbf{S}}_{\chi} \cdot \frac{\hat{\mathbf{q}}}{m_{N}}\right)\left[\left(\hat{\mathbf{S}}_{N} \times \hat{\mathbf{v}}^{\perp}\right) \cdot \frac{\hat{\mathbf{q}}}{m_{N}}\right]$ \\
\hline
\end{tabular}

Table 1: The 14 leading order WIMP-nucleon interaction operators.

\section{WIMP capture by the Sun}

This section contains the mathematical background for the WIMP capture process. The first subsection present the equations that govern capture under the assumptions of a cold Sun and intantaneous thermalization to a thermal density profile. In the second subsection the assumption of a cold Sun is relaxed, in order to properly model a WIMP's thermalization trajectory in the Sun.

\subsection{Capture and annihilation}

Collisions with solar nuclei cause halo WIMPs to lose kinetic energy, potentially binding them to the Sun's gravity. The probablity per unit time for a scattering event that results in a WIMP velocity below the local escape velocity $v(r)$ is $[9,12]$

$$
\Omega_{v}^{-}(w)=\sum_{T} n_{T} w \Theta\left(\frac{\mu_{T}}{\mu_{+, T}^{2}}-\frac{u^{2}}{w^{2}}\right) \int_{E_{k} u^{2} / w^{2}}^{E_{k} \mu_{T} / \mu_{+, T}^{2}} \mathrm{~d} E_{r} \frac{\mathrm{d} \sigma\left(E_{r}, w^{2}\right)}{\mathrm{d} E_{r}},
$$

where $m_{\chi}\left(m_{T}\right)$ is the dark matter (target nucleus) mass, $n_{T}$ is the target species number density, $u$ and $w=\sqrt{u^{2}+v(r)^{2}}$ are the dark matter particle velocities at point of scatter and at infinite radius, and $\mathrm{d} \sigma / \mathrm{d} E_{r}$ is the differential cross section. The integral bounds correspond to the minimal energy transfer necessary for capture, and the highest possible energy transfer in an elastic collision, given by the WIMP's kinetic energy $E_{k}=m_{\chi} w^{2} / 2$, and dimensionless parameters $\mu_{T}=m_{\chi} / m_{T}$ and $\mu_{+, T}=\left(\mu_{T}+1\right) / 2$. The Heaviside function, $\Theta$, ensures that this integral is positive.

The WIMP capture rate per volume is given by

$$
\frac{\mathrm{d} C_{c}}{\mathrm{~d} V}=\int_{0}^{\infty} \mathrm{d} u \frac{f(u)}{u} w \Omega_{v}^{-}(w),
$$

where $f(u)$ is the WIMP halo velocity distribution. The total capture rate is found by integrating this expression over the Sun's volume.

Captured WIMPs settle to thermal equibrium with the Sun's core, where they can annihilate to Standard Model particles. The value for the thermally averaged annihilation cross-section used 
in this work is $\left\langle\sigma_{A} v\right\rangle \simeq 2 \times 10^{-26} \mathrm{~cm}^{3} \mathrm{~s}^{-1}$ [13]. The total number of WIMPs annihilated per unit time is $C_{a} N^{2}$, where $N$ is the total number of trapped WIMPs and $C_{a}$ is given by

$$
C_{a}=\frac{4 \pi\left\langle\sigma_{A} v\right\rangle}{N^{2}} \int_{0}^{R_{\odot}} \varepsilon^{2}(r) r^{2} \mathrm{~d} r
$$

In this expression, $\varepsilon(r)$ is the WIMP number density function. It is commonly assumed that thermalizaed WIMPs follow a thermal profile,

$$
\varepsilon(r) \propto \exp \left(-\frac{m_{\chi} \phi(r)}{k_{B} T_{c}}\right),
$$

determined by the Sun's core temperature $T_{c}$ and gravitational potential $\phi(r)$.

Assuming a negligible thermalization time, the amount of WIMPs trapped within the Sun, $N$, is described by the following differential equation,

$$
\frac{\mathrm{d} N}{\mathrm{~d} t}=C_{c}-C_{a} N^{2}
$$

with solution

$$
N(t)=\sqrt{\frac{C_{c}}{C_{a}}} \tanh \left(\sqrt{C_{c} C_{a}} t\right) .
$$

\subsection{WIMP trajectories}

The thermalization process has been studied by means of Monte-Carlo integration, by following WIMP trajectories from the first scattering event that results in capture, to having reached thermal equilibrium after subsequent collisions.

A WIMP's orbit around a spherically symmetric massive body can be parameterized by its energy $E$ and angular momentum $J$. The WIMP-nucleus interactions are weak and the Sun is optically thin. When neglecting any thermal motion of the target nuclei, the probability of scatter in a thin shell of thickness $\mathrm{d} r$ during one orbital period is

$$
\mathrm{d} P_{s c}=\mathrm{d} s n_{T} \int_{0}^{E_{k}(w) \mu_{T} / \mu_{+, T}^{2}} \mathrm{~d} E_{r} \frac{\mathrm{d} \sigma\left(E_{r}, w^{2}\right)}{\mathrm{d} E_{r}} .
$$

By relaxing the assumption of a cold Sun, the scattering probability per orbital period is instead

$$
\mathrm{d} P_{s c}=\frac{\mathrm{d} s n_{T}}{w} \int_{-\infty}^{\infty} \mathrm{d} \tilde{w} \int_{0}^{E_{k}(\tilde{w}) \mu_{T} / \mu_{+, T}^{2}} \mathrm{~d} E_{r} \frac{\mathrm{d} \sigma\left(E_{r}, \tilde{w}^{2}\right)}{\mathrm{d} E_{r}} f_{T}(\tilde{w}) \tilde{w},
$$

where $\tilde{w}$ is the collisional velocity between the WIMP and target nucleus, and $f_{T}(\tilde{w})$ is the velocity distribution of a nucleus in the WIMP rest frame, assumed to be a thermal Maxwell-Boltzmann distribution boosted by a velocity $w$.

Even if the WIMP velocity and collisional velocity are known, the thermal velocity of the nuclei, $v_{t h}$, is not. Its component along the axis of the WIMP's motion, $v_{t h z}$, is limited to values $w-\tilde{w} \leq v_{t h z} \leq w+\tilde{w}$. The perpendicular component of the thermal velocity must fulfill that $v_{t h \perp}^{2}+$ $\left(v_{t h z}-w\right)^{2}=\tilde{w}^{2}$. Wuth these conditions, the probability density function for $v_{t h z}$ becomes 


$$
\tilde{f}_{T}\left(v_{t h z}\right) \mathrm{d} v_{t h z}=2 \pi \tilde{w}\left(\frac{m_{T}}{2 \pi k_{B} T}\right)^{3 / 2} \exp \left(-\frac{m_{T}}{2 k_{B} T}\left(\tilde{w}^{2}-w^{2}+2 w v_{t h z}\right)\right) \mathrm{d} v_{t h z} .
$$

Integrating this function over the range of possible $v_{t h z}$ gives the boosted Maxwell-Boltzmann distribution. After finding the collisional velocity, thermal velocity and recoil energy of a collision, there are two remaining degrees of freedom. They are found in the angular orientation of $v_{t h \perp}$ and in one scattering angle, both of which are trivially randomized in the rest frame of the target nucleus where all angles are equiprobable.

\section{Results}

Thermalization time scales and subsequent thermal density profiles are calculated by sampling a large number of WIMP trajectories. The considered WIMP is in the mass range of 10-1000 $\mathrm{GeV}$ and spin $1 / 2$. The WIMP halo velocity distribution is assumed to be a Maxwell-Boltzmann distribution with velocity dispersion of $270 \mathrm{~km} / \mathrm{s}$, a Local Standard of Rest velocity of $220 \mathrm{~km} / \mathrm{s}$, and a local dark matter density of $0.4 \mathrm{GeV} / \mathrm{cm}^{3}$.

The distribution of thermalized WIMPs have been assesed by following a single WIMP's trajectory, starting from a state of thermal equilibrium, as it collides with atomic nuclei within the solar medium. It was found to adhere to a thermal profile described by equation 3.4 for all WIMP-nucleon interaction operators of table 1 . This is shown in figure 1 for operators $\hat{\mathscr{O}}_{1}$ and $\hat{\mathscr{O}}_{15}$.

The thermalization time medians for all operators of table 1 are visible in figure 2 . In this figure, the coupling constants are normalized to values such that the WIMP-nucleon cross section at collisional velocity $1000 \mathrm{~km} / \mathrm{s}$ is $\sigma_{\chi N}=10^{-44} \mathrm{~cm}^{2}$. The longest thermalization times are from operators that scatter almost exclusively on hydrogen, which is the case for operators $\hat{\mathscr{O}}_{4}, \hat{\mathscr{O}}_{7}$ and the isovector component of $\hat{\mathscr{O}}_{1}$, and in the higher mass range also $\hat{\mathscr{O}}_{11}, \hat{\mathscr{O}}_{12}$ and the isoscalar component of $\hat{\mathscr{O}}_{1}$. Other operators also interact with heavier nuclei and actually spend the majority of the thermalization time in the solar interior. Due to the inverse proportionality of thermalization time and WIMP-nucleon cross section, multiplying the values in 2 by a factor $2.2 \times 10^{-54} \mathrm{~cm}^{2} / \mathrm{yr}$ gives the cross section for which the thermalization time is equal to the age of the Sun. In order to safely assume instaneous thermalization, a margin of at least one order of magnitude would be preferred.

In order to detect a high-energy neutrino signal coming from the Sun, the capture rate must be high enough for annihilation to have come into significant effect. Because the annihilation rate and the neutrino signal is proportional to the WIMP density squared, significant annihilation presupposes that the number of trapped WIMPs is close to its equilibrium amount. In figure 3, the thermalization time medians are presented for operator coupling constants that are normalized such that the total capture rate is $C_{c}=1 /\left(C_{a} t_{\odot}^{2}\right)$, where $t_{\odot}$ is the age of the Sun. By using this value for $C_{c}$, the number of annihilation events per unit time is $\tanh ^{2}(1) \simeq 58 \%$ of its equilibrium value. The thermalization time scales for a specific WIMP mass differs at most $\sim 3$ orders of magnitude between operators.

Conclusions are found in the abstract. 

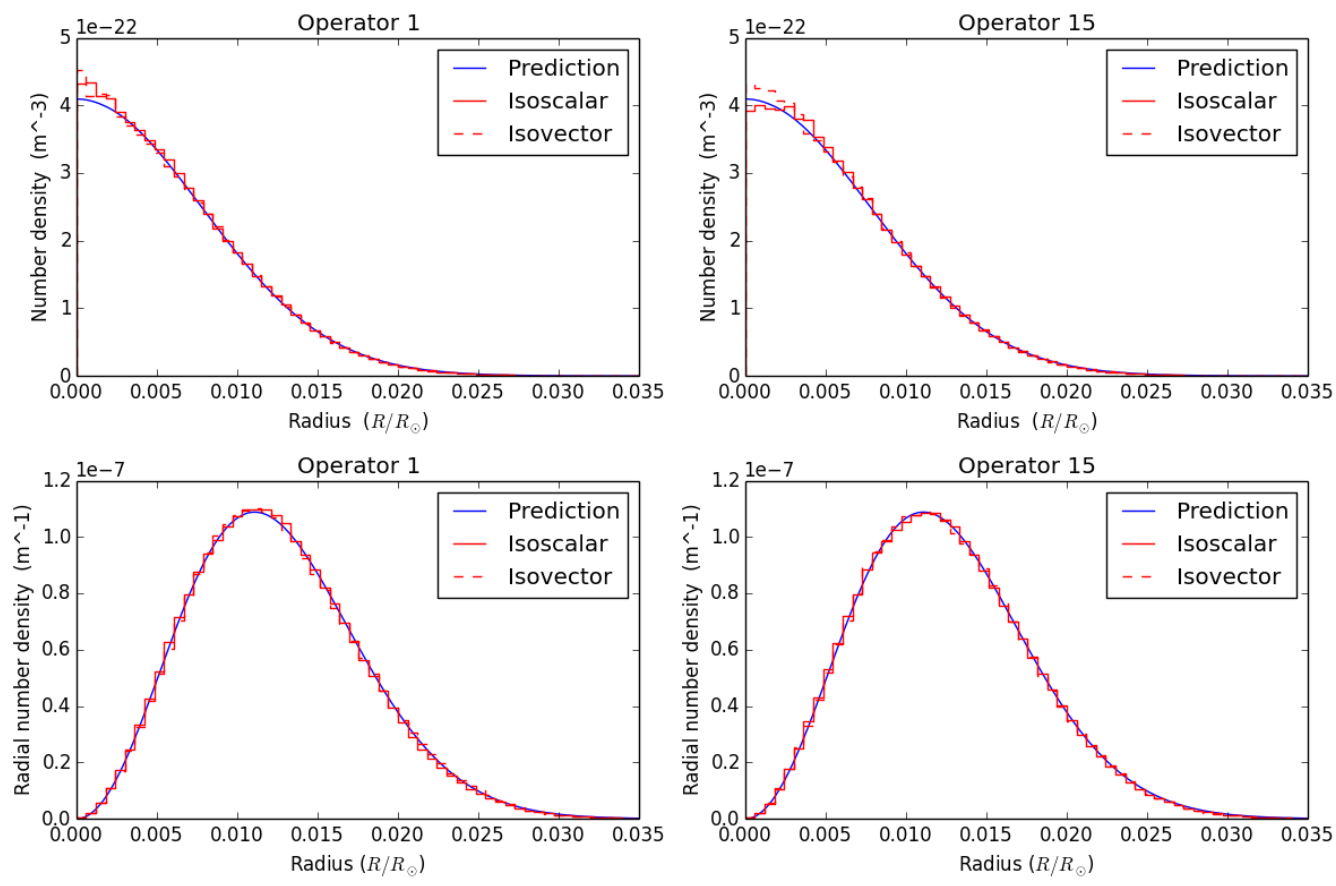

Figure 1: The density profile of thermalized WIMPs are shown for a $100 \mathrm{GeV}$ WIMP, with WIMP-nucleon interaction operators $\hat{\mathscr{O}}_{1}$ (left) and $\hat{\mathscr{O}}_{15}$ (right), with isoscalar (solid red) and isovector (dashed red) coupling. The thermal profile (solid blue) is thermal distribution of equation 3.4. The upper panels show the number density as a function of radius; the lower panels show the probability for a WIMP to be located at radius $R$ at a given time.

\section{References}

[1] A. Widmark, Thermalization time scales for WIMP capture by the Sun in effective theories, JCAP 1705 (2017) 046 [arXiv: 1703.06878 ].

[2] G. Jungman, M. Kamionkowski and K. Griest, Supersymmetric Dark Matter, Phys.Rept. 267 (1996) 195-373 [hep-ph/9506380].

[3] L. Bergström, Non-Baryonic Dark Matter: Observational Evidence and Detection Methods, Rept.Prog.Phys. 63 (2000) 793-841 [hep-ph/ 0002126 ].

[4] J. Silk, K. Olive and M. Srednicki, The photino, the sun, and high-energy neutrinos, Phys.Rev.Lett. 55 (1985) 257-259.

[5] W.H. Press and D.N. Spergel, Capture by the sun of a galactic population of weakly interacting, massive particles, ApJ 296 (1985) 679-684.

[6] Z.-L. Liang, Y.-L. Wu, Direct detection and solar capture of spin-dependent dark matter, Phys.Rev. D89 (2014) 013010 [arXiv: 1308 . 5897].

[7] W.-L. Guo, Z.-L. Liang and Y.-L. Wu, Direct detection and solar capture of dark matter with momentum and velocity dependent elastic scattering, Nucl.Phys. $\mathbf{B 8 7 8}$ (2014) 295-308 [arXiv:1305.0912].

[8] J. Blumenthal, P. Gretskov, M. Krämer and C. Wiebusch, Effective field theory interpretation of searches for dark matter annihilation in the Sun with the IceCube Neutrino Observatory, Phys.Rev. D91 (2015) 035002 [arXiv: 1411.5917 ]. 

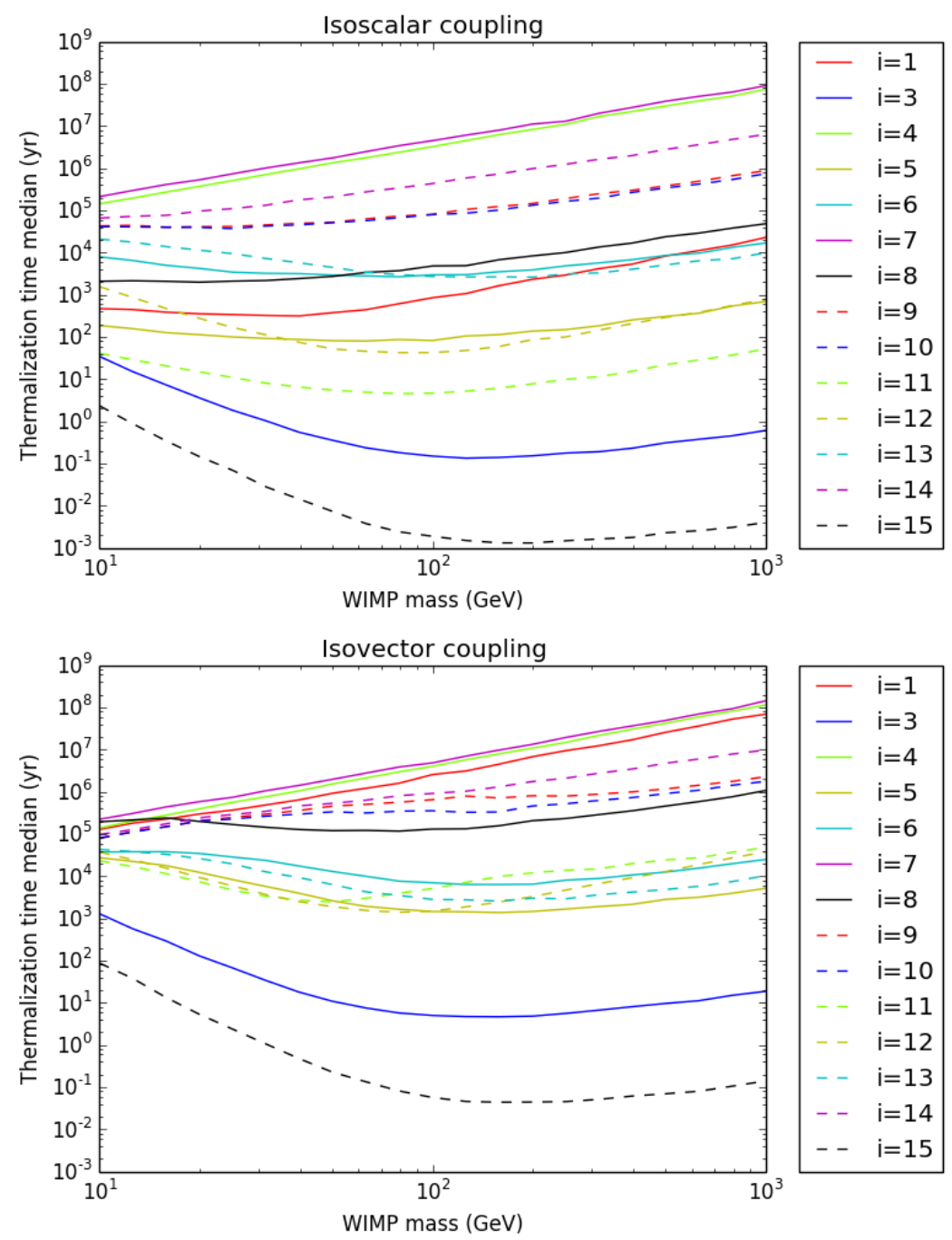

Figure 2: Thermalization time medians for operators $\hat{\mathscr{O}}_{i}$, with isoscalar (upper panel) and isovector (lower panel) couplings. The WIMP-nucleon cross section at collisional velocity $1000 \mathrm{~km} / \mathrm{s}$ is set to $\sigma_{\chi N}=10^{-44}$ $\mathrm{cm}^{2}$.

[9] R. Catena and B. Schwabe, Form factors for dark matter capture by the Sun in effective theories, JCAP 1504 (2015) 042 [arXiv: 1501 . 03729].

[10] R. Catena and A. Widmark, WIMP capture by the Sun in the effective theory of dark matter self-interactions, JCAP 1612 (2016) 016 [arXiv: 1609 . 04825].

[11] A.L. Fitzpatrick, W. Haxton, E. Katz, N. Lubbers and Y. Xu, The Effective Field Theory of Dark Matter Direct Detection, JCAP 1302 (2013) 004 [arXiv : 1203.3542 ].

[12] A. Gould, Resonant Enhancements in Weakly Interacting Massive Particle Capture by the Earth, ApJ 321 (1987) 571-585.

[13] G. Steigman, B. Dasgupta and J.F. Beacom, Precise Relic WIMP Abundance and its Impact on Searches for Dark Matter Annihilation, Phys.Rev. D86 (2012) 023506 [arXiv : 1204 . 3622 ]. 

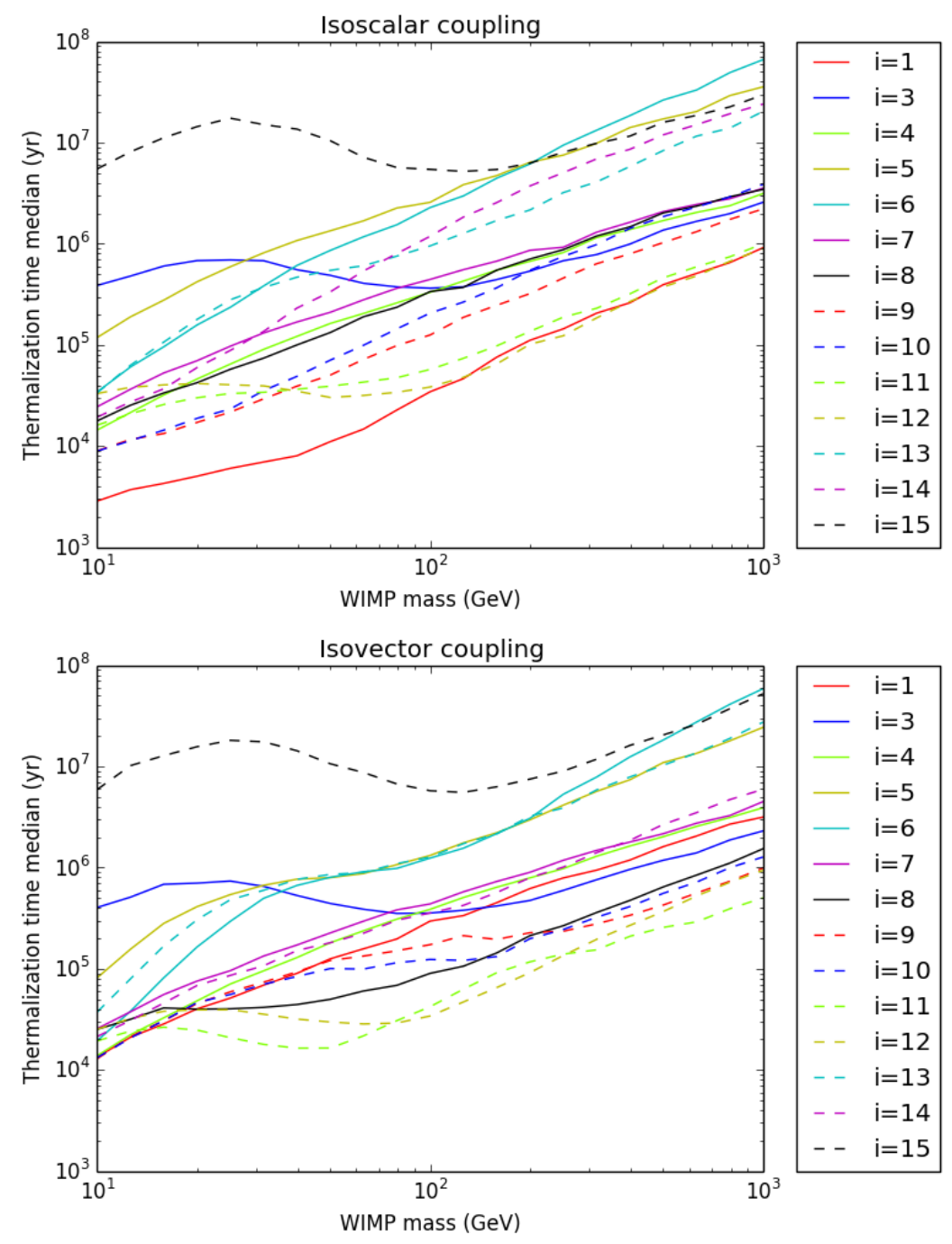

Figure 3: Thermalization time medians for operators $\hat{\mathscr{O}}_{i}$, with isoscalar (upper panel) and isovector (lower panel) couplings. The coupling strength is normalized to a capture rate, $C_{c}=1 /\left(C_{a} t_{\odot}^{2}\right)$. Using this capture rate and the current age of the solar system, significant annihilation has come into effect. 